\title{
Gamificación en la estimulación cognitiva de niños entre 5-7 años con síndrome de down en la UNAE
}

\author{
Erika Daniela Balarezo Lata \\ edbalarezo@sudamericano.edu.ec \\ David Israel Mendieta Parra \\ dimendieta.2@sudamericano.edu.ec
}

Juan Marcelo Pérez Pérez jperez@sudamericano.edu.ec

Galo Patricio Hurtado Crespo

gphurtado@sudamericano.edu.ec

Instituto Superior Tecnológico Particular Sudamericano Cuenca, Ecuador

\section{RESUMEN}

La investigación busca analizar el uso de la gamificación en niños con Síndrome de Down entre 5-7 años, La innovación que se planteará servirá para fortalecer la estimulación cognitiva, memoria y percepción individual de cada niño. Las herramientas del uso de las TIC'S en la educación se consideran como una novedosa opción el uso distintos recursos virtuales para fortalecer el aprendizaje significativo. Se considera que, en la educación, el método del juego es necesario para despertar la motivación intrínseca de los estudiantes y así lograr avances en el proceso de enseñanza-aprendizaje y de esta manera, los estudiantes tendrán mejores resultados académicos. Se recopilo opiniones de docentes especializados en la rama de Educación Especial, quienes consideran como una buena opción de aprendizaje la propuesta de gamificación en niños con Síndrome de Down, los niños con discapacidad intelectual pueden adquirir diversas habilidades que les servirían en su vida cotidiana. A través de las diversas metodologías didácticas, una de estas la gamificación. Con la propuesta sea de paso al uso de recursos educativos digitales en todo el ámbito escolar; enfocados en acoplarse en las necesidades individuales de cada niño especialmente en niños con Síndrome de Down entre otras necesidades educativas especiales asociadas a la discapacidad.

Palabras clave: gamificación; estimulación cognitiva; síndrome de down; enseñanzaaprendizaje 


\title{
Gamification in the cognitive stimulation of children between 5-7 years old with down syndrome at UNAE
}

\begin{abstract}
The research seeks to analyze the use of gamification in children with Down Syndrome between 5-7 years old. The innovation that will be proposed will serve to strengthen cognitive stimulation, memory and individual perception of each child. The use of ICT tools in education is considered as a novel option the use of different virtual resources to strengthen meaningful learning. It is considered that, in education, the game method is necessary to awaken the intrinsic motivation of students and thus achieve progress in the teaching-learning process and in this way, students will have better academic results. We gathered opinions of teachers specialized in the field of Special Education, who consider the gamification proposal as a good learning option for children with Down Syndrome, children with intellectual disabilities can acquire various skills that would serve them in their daily lives. Through various didactic methodologies, one of these is gamification. With the proposal is to move to the use of digital educational resources throughout the school environment; focused on coupling in the individual needs of each child especially in children with Down syndrome among other special educational needs associated with disability.
\end{abstract}

Keywords: gamification; cognitive stimulation; down's syndrome; teaching-learning

Artículo recibido: 15 enero 2022 Aceptado para publicación: 08 febrero 2022 Correspondencia: edbalarezo@ sudamericano.edu.ec Conflictos de Interés: Ninguna que declarar 


\section{INTRODUCCIÓN}

La educación especial es un continuo de beneficios educativos, que incluyen una combinación de servicios, técnicas, estrategias, conocimientos y recursos educativos, diseñado para garantizar un proceso de aprendizaje inclusivo, flexible, dinámico para las personas. Aquellos con necesidades educativas especiales, temporales o permanentes, provistas por Organizaciones específicas de apoyo diverso. (Narvaja, 2018). Según datos del Consejo Nacional para la igualdad de Discapacidades en sus estadísticas de discapacidad; registra en la provincia de Cañar; cantón Azogues un total de 93 personas con discapacidad; distribuyéndose de la siguiente manera 32,26\% (30 personas) mujeres entre la edad de 4 a 12 años, y 67.74\% (63 personas) hombres entre la edad de 4 a 12 años; que presentan distintos tipos de discapacidad entre estas: discapacidad intelectual que con porcentaje de 83,87\% (78 personas), discapacidad psicosocial 16,13\% (15 personas). Según el autor menciona que existen diversos grados de discapacidad en este caso los datos presentados corresponden del 30 al 49 por ciento ubicándose en un grado de discapacidad medio (Consejo Nacional para la Igualdad de Discapacidades, 2021).

La propuesta de gamificación en la metodología de enseñanza-aprendizaje para la estimulación cognitiva de niños con síndrome de Down será una nueva propuesta de innovación para los padres, docentes, cuidadores y niños, siendo más accesible y fácil de entender; con juegos basados en percepción, atención, memoria, lenguaje. El cambio constante se produce en el mundo de la tecnología, debe avanzar continuamente con la realidad educativa que se vive actualmente, una realidad virtual que debe acoplarse a la necesidad individual de estudiantes, particularmente en niños con síndrome de Down, considerando que requieren mayor apoyo en cuanto su proceso de enseñanza-aprendizaje; con actividades cotidianas.

Debido a la situación que ha generado el confinamiento y el cambio en la educación, en especial en la educación de niños con síndrome de Down, se considera oportuno la propuesta de gamificación que contribuya la mejora continua de la estimulación en distintas áreas de la formación y desarrollo del mismo; que sea de fácil acceso y accesible para las personas involucradas en el tema de investigación debido a las condiciones académicas que atraviesan. 
La investigación está centrada en conocer las distintas dificultades durante el proceso de enseñanza-aprendizaje de niños con síndrome de Down. Tiene como objetivo cubrir las necesidades que se han presentado a raíz de la situación de la pandemia, tanto en la educación, formación y desarrollo de los niños con síndrome de Down.

Con la investigación se pretende llegar a un número considerable de beneficiarios; partiendo de los niños y niñas del cantón Azogues; en vinculación con la Universidad Nacional de Educación (UNAE), quien según datos oficiales Consejo Nacional para la igualdad de Discapacidades existe un total de 93 personas con discapacidad intelectual, que corresponde a un 25,68\% de personas con discapacidad en dicho cantón. Para aprovechar al máximo las distintas capacidades de la tecnología, mejorando la calidad educativa, el avance individual de cada uno de los niños, con distintos prototipos de juegos, actividades virtuales, y tareas que se acoplen a cada uno.

La discapacidad intelectual es un trastorno del neurodesarrollo que se caracteriza por deficiencias cognitivas y cambios funcionales en la conducta adaptativa que surgen durante su desarrollo. Cabe señalar que esto produce una multiplicidad de síntomas, es considerado por la neuropsicología como el eje de análisis. En este sentido, se enfatizará por qué es necesario un examen neuropsicológico en esta condición. Esta asociación incluye, por un lado, determinar si la discapacidad del niño se debe a una alteración del sistema nervioso o a condiciones desfavorables del entorno que se desenvuelve. El examen neuropsicológico permite saber qué región del cerebro es responsable de una discapacidad u otra. Además, se pueden identificar características únicas del desarrollo del niño, es decir, el perfil de logros del niño (fortalezas y debilidades), y no necesariamente establecer un diagnóstico de discapacidad intelectual, y sobre esta base establecer intervenciones adaptadas a las características y necesidades de cada uno (Luis M. Echavarría-Ramírez, 2021).

A muchos niños y niñas les resulta difícil aprender de diferentes maneras. A veces puede ser una dificultad específica, como cuando un niño tiene dificultades con una tarea específica como leer o escribir, pero otras veces puede ser una dificultad general, como cuando aprende más lentamente de lo habitual durante una serie de tareas o cuando enfrentado a la dificultad. observado en muchas áreas del desarrollo de un niño. Sin embargo, la división entre problemas de aprendizaje generales y específicos no es tan sencilla, por el motivo que los estudiantes tienen una dificultad particular a menudo, 
tienen dificultades en muchas áreas sin presentar necesariamente los problemas en la mayoría de las áreas de desarrollo. La discapacidad intelectual es una de las más comunes entre las dificultades generales o globales en el aprendizaje y el desarrollo académico. Tradicionalmente, los estudiantes con estos problemas han sido denominados colectivamente retrasados en el desarrollo o retrasados. Para algunos niños, los problemas no se hacen evidentes hasta que comienzan la escuela y su progreso se compara con el de sus compañeros. Los estudiantes con problemas generales de desarrollo tienen tasas de aprendizaje más lentas y umbrales más bajos. Un techo más bajo indica un nivel limitado de desarrollo cognitivo; por lo que existen límites superiores para el rendimiento. Por este motivo, la discapacidad intelectual se clasifica como un problema global o trastorno del desarrollo o retraso del niño, aunque las particularidades de este problema son específicas y específicas de cada caso (Rocío \& Peredo Videa, 2016).

En la actualidad existe una gran variedad de metodologías para la enseñanza-aprendizaje en distintos ámbitos, en especial escenarios virtuales y tecnológicos, que han replanteado el uso de diversas estrategias, metodologías y materiales en el proceso de enseñanzaaprendizaje de niños con síndrome de Down, partiendo del uso de dispositivos audiovisuales y conectividad que no siempre están al alcance de muchos o en ocasiones son muy difíciles de manejar para los padres de familia y docentes (José Flores Tena \& Tena, 2021).

Se propone realizar una revisión sistemática enfocada en la creación y posterior uso de un prototipo de gamificación en la metodología de enseñanza-aprendizaje para la estimulación cognitiva de niños entre 5-7 años con síndrome de Down. Es importante recopilar la información en gestores documentales para validar el prototipo de gamificación que ayude a la estimulación cognitiva de niños con síndrome de Down; Con la presente investigación y revisión sistemática de información, datos, se presenta una idea más clara sobre la gamificación, que favorezcan con el avance del proceso de enseñanza-aprendizaje, en niños y niñas con diferentes barreras en el ámbito educativo.

Se toma en cuenta el uso de 3 variables que parten desde la gamificación la misma que hace referencia a una técnica de aprendizaje usada en las aulas de clases, apoyada en emplear una gran variedad de recursos y herramientas que ayudan a los docentes a personalizar las actividades individuales de cada uno de los niños, esta última, suma importancia al objetivo de la presente investigación que es individualizar las actividades 
a cada niño, dependiendo de su avance y capacidad cognitiva, la gamificación, además de las consideraciones a tomarse por parte del docente guía y padres de familia. Así pues, mencionamos a Zapata Z (2019) quien afirma que la técnica de aprendizaje mediante la gamificación se "traslada la mecánica de los juegos al ámbito educativo-profesional con el fin de conseguir mejores resultados, absorber mejor algunos conocimientos, mejorar alguna habilidad, o bien recompensar acciones concretas, entre otros muchos objetivos" (p. 10), es decir la gamificación en las instituciones educativas a nivel general, es considerada de manera favorable; porque la misma ofrece distintas maneras de trabajo personalizado, es importante recalcar que se debe partir del desarrollo evolutivo o cognitivo del niño, es decir partir de conceptos definidos, para así poderlos implementar en la gamificación

La gamificación desarrolla escenarios favorables al aprendizaje de los estudiantes, promueve su participación, un rol activo a docentes, estudiantes asociados al mecanismo de enseñanza-aprendizaje, debido a las dificultades surgidas en cada rol de transmisión para asimilarlo respectivamente, planteando exigencias. Para implementar métodos de enseñanza -aprendizaje hacia escenarios lúdicos y atractivos, creando entusiasmo y motivación a los estudiantes, la alternativa de la gamificación aparece en el campo de la educación, incluye la aplicación de estrategias, pensamiento y mecánicas de jugar, con el propósito de hacer que las personas adopten ciertos comportamientos.

En relación con el proceso de enseñanza-aprendizaje que está estrictamente vinculado con la gamificación en la educación, es necesario mencionar puntos importantes que se mantendrá para la presente investigación. Tal como lo menciona Pimienta HJ (2016) “Las estrategias de enseñanza aprendizaje son instrumentos al docente para contribuir a la implementación y desarrollo de las competencias de los estudiantes". Con base en una secuencia didáctica que incluye inicio, desarrollo y cierre, es conveniente utilizar estas estrategias de forma permanente tomando en cuenta las competencias específicas para contribuir al desarrollo.

La enseñanza no puede entenderse más que en relación al aprendizaje; y esta realidad relaciona no sólo a los procesos vinculados a enseñar, sino también a aquellos vinculados a aprender. Se destaca la definición de método, así como una clasificación en función del grado de actividad del profesor y de la independencia de los estudiantes siendo de las mismas clasificaciones de método de enseñanza-aprendizaje (Gerardo Meneses Benítez, 
2016). Relaciona las diferentes concepciones didácticas con los procesos de enseñanzaaprendizaje que generan: la comunicación y el currículum. Se trata de tres maneras de entender las relaciones entre docente, contenidos, estrategias y prácticas.

En relación a la gamificación en la educación esta va de la mano con la estimulación cognitiva; que engloba varias actividades que están dirigidas a optimizar las distintas capacidades, el rendimiento y eficacia en las funciones. La estimulación cognitiva actúa sobre aquellas capacidades y habilidades que se encuentran aún preservadas por medio de actividades y programas que promueven su mejora y potenciación por mecanismos de neuro plasticidad cerebral. Así como lo interpretan (Espert Tortajada \& Villalba, 2015). Quienes definen a la estimulación cognitiva como "el conjunto de técnicas y estrategias que pretenden optimizar la eficacia del funcionamiento de las distintas capacidades y funciones cognitivas (percepción, atención, razonamiento, abstracción, memoria, lenguaje, procesos de orientación y praxias) mediante una serie situaciones y actividades concreta" (p. 74), las actividades centradas en mejorar las capacidades individuales de los niños con o sin alguna barrera en el aprendizaje, de tal manera que las actividades sean de una manera significativa en su avance escolar y personal.

En relación con los epígrafes anteriormente abordados mantiene una estrecha relación con el término Síndrome de Down, que comúnmente refiere a un trastorno genético que se origina con la división celular anormal que produce una copia adicional total o parcial en el cromosoma 21, que provoca cambios o alteraciones en el desarrollo y características físicas y en ocasiones intelectuales, es decir que pueden presentar TDA (Trastorno por déficit de atención) o problemas en el avance de su educación. Es importante mencionar que las barreras en el aprendizaje que presentan los niños con síndrome de Down, no comprometen sus actividades en su vida diaria o su independencia como personas, el objetivo del presente proyecto es realizar una gamificación, es decir una actividad virtual centrada en la educación que estimule cognitivamente las capacidades, como la percepción, atención, razonamiento, abstracción, memoria y lenguaje en niños con Síndrome de Down, basados en el desarrollo evolutivo de niños entre 5-7 años, se tiene como presente desarrollar, motivar y avanzar significativamente en su proceso de enseñanza-aprendizaje. Es importante mencionar que el objetivo final que menciona (Ruiz Rodríguez, 2009). Es que cualquier intervención con personas con discapacidad, 
como con cualquier persona, la inclusión, y la participación en la sociedad como ciudadanos es un pleno derecho.

La estimulación temprana promueve el desarrollo óptimo de los sentidos y las habilidades de los niños en los primeros años, también proporciona una variedad de actividades científicas y de aplicaciones, es importante asistir a las capacidades de los niños en cada etapa de su vida diaria, aplicando técnicas completas y agradables a los bebés para mejorar las habilidades cognitivas, los objetivos deben exceder la capacidad de cada niño donde comienza a entrenar y crear su propia conexión para controlar el cerebro con el cuerpo. Se proporcionará a los niños una estimulación temprana adecuada (Kevin \& Cruces Geraldine, 2021).

La inclusión educativa es un factor sumamente importante a ser considerado por todos los actores del sistema educativo, en los diferentes niveles es necesario analizar cómo se hace, sin excluir a nadie, porque también en una escuela, es un espacio de co-construcción en las relaciones interpersonales, donde se fomenta el respeto y la aceptación mutua, situación no sólo beneficiosa para los incluidos sino también para los que forman parte de este proceso holístico. En Ecuador, el tema de la inclusión cobra cada vez más importancia, se aborda desde aspectos sociales, laborales y educativos (Jiménez, 2019). La estrategia de enseñanza-aprendizaje es una herramienta utilizada por los maestros para ayudar a implementar, desarrollar las habilidades de los estudiantes. Con base en la secuencia de enseñanza que incluye inicio, desarrollo final, considerando las habilidades específicas que se pretenderá ayudar a desarrollar, es conveniente utilizar estas estrategias de manera permanente (Vargas-Murillo, 2020). Existen estrategias para recopilar conocimientos previos y organizar o construir contenidos.

El uso correcto de estas estrategias puede promover el recuerdo de explorar conocimientos previos, ayuda a iniciar las actividades en la secuencia de enseñanza. Son importantes como fuente de organización gráfica de conocimientos descubiertos, algo muy útil para estudiantes cuando necesitan tomar notas. Es impulsado por puentes cognitivos entre lo que el sujeto ya sabe.

El desarrollo verdadero que necesita saber y asimilar significativamente es conocer una nueva zona de crecimiento proximal que conduce al potencial de crecimiento. Los puentes son formados por organizadores anteriores, es decir, conceptos, ideas originales y documentos introductorios, presentados como marco de referencia para los nuevos 
conceptos y relaciones. La clave para el aprendizaje significativo radica en la relación entre el material nuevo y las ideas existentes en las estructuras cognitivas de los estudiantes. Por lo tanto, la efectividad de tal aprendizaje depende de su significado, no de las técnicas de memorización.

El proceso de aprendizaje pedagógico converge objetivos de aprendizaje, contenido, Estrategias de aprendizaje y enseñanza, formularios de evaluación y procesos. La tutoría es necesaria para conocer el estilo de aprendizaje y enseñanza y la motivación, la innovación educativa y creativa es concreta, que es un proceso complejo multifactorial, por lo que también es una gran cantidad de definiciones. La estrategia educativa y su integración con tecnologías digitales para fortalecer el proceso de aprendizaje pedagógico educativo, debido a muchas funciones y condiciones, el proceso de enseñanza es muy complicado; hay que pensar al revés para darle una modificación y transformación a favor de su desarrollo. La historia de la educación muestra que hubo intentos de diseñar procesos educativos para corresponder a las condiciones históricas específicas de la época a la que se refiere y a las posibilidades que el desarrollo de la ciencia ofrece para la actualidad. Los modelos están pensados para acomodar diferentes niveles de materialización y también diferentes partes del proceso educativo, cada modelo educativo se basa en modelos psicológicos del proceso de aprendizaje, en modelos sociológicos, comunicativos, ecológicos o sensoriales a partir de los cuales se necesita esta relación para orientar adecuadamente la investigación y renovar modelos educativos. El modelado de procesos educativos tiene peculiaridades propias que hacen que su modelo sea diferente a otros.

La formulación del concepto del modelo educativo facilitará la identificación, evaluación y desarrollo de modelos educativos para lograr el nuevo efecto educativo (Ortiz Ocaña, 2013). Se centra en las estrategias que los estudiantes utilizan en su entrenamiento académico como comprobar, construir, organizar y comprender.

Con respecto a la reducción de la división digital en el contexto de la educación. Finalmente, se enfatiza en la integración estratégica educativa y una nueva información tecnológica de comunicación disponible, y así, implican abrir nuevos enlaces dinámicos de interacción entre maestros y estudiantes, la reducción de la división digital en el contexto de la educación se enfatiza en la integración. La comprensión es un proceso en el que las claves proporcionadas por el autor y los conocimientos previamente poseídos 
por el niño se utilizan para inferir el significado del texto, que es la base para el desarrollo de las habilidades y destrezas de los niños (Federico Cintrón Fiallo, 2004).

El término estrategia se utiliza cada vez más en la literatura educativa, a pesar de sus múltiples significados e interpretaciones. Las ventajas que puede traer su uso adecuado en los procesos educativos son indiscutibles, frente a un mundo en constante cambio, la educación continua como una respuesta educativa estratégica para equipar a los estudiantes con las herramientas intelectuales que les permitan adaptarse, trabajar y expandir el conocimiento (Ximena Patricia Curay Correa \& Diego Esteban Fernández Olivo, 2021). Es necesario planificar y utilizar estrategias de enseñanza que promuevan el aprendizaje reflexivo y la educación para enfrentar los cambios, la incertidumbre y la dinámica del mundo actual. Cuando abordamos el estudio de las estrategias de enseñanza y los métodos de enseñanza-aprendizaje, es necesario tener precisión conceptual desde el inicio, por la falta de criterios unificados en la conceptualización de los científicos. Al respecto, es necesario considerar brevemente algunos de sus significados y explicar los significados que se le dan a cada uno de ellos, sin buscar agotar la variedad de posiciones al respecto.

Según (Zúñiga et al., 2020) menciona sobre la capacidad de la configuración en las pequeñas organizaciones de desarrollo de software es el resultado de un proyecto a través de cuestionarios de aplicación, se encontró que las organizaciones pequeñas complementan el proceso de desarrollo de software con la gestión de la configuración. Debido a la necesidad de actualizar siempre los cambios de configuración de los productos de software. La transformación digital ha afectado a las pequeñas organizaciones, automatizando parte de sus procesos, incluyendo el apoyo a la gestión de la configuración con capacidades de nivel 1. Para comprender las realidades de las organizaciones desde un punto de vista En este caso, se utilizó el modelo CMMI 2.0 de capacidades para evaluar las capacidades del Área de Procesos de Gestión de la Configuración. Las técnicas utilizadas para la recolección de datos son: Entrevistas y encuestas, debido a la variedad de contextos en los que se desarrollan las organizaciones estudiadas.

\section{ESTRATEGIAS METODOLÓGICAS O MATERIALES Y MÉTODOS}

Se utilizará como técnica principal la observación directa es un método de recopilación de datos que esencialmente incluye la observación del objeto de investigación en una 
situación específica. Todo esto se hace sin necesidad de intervenir o modificar el entorno en el que se produce el objeto. De lo contrario, los datos obtenidos no serán válidos. Los métodos de recolección de datos se utilizan en los casos en que otros sistemas, como encuestas, cuestionarios, entre otros, no son tan efectivos. a niños con Síndrome de Down y sus familiares cercanos o tutores. Así también aplicando la observación participante, como una forma de observación cualitativa, permite una mejor comprensión de lo que está sucediendo en el entrenamiento. De alguna manera, se vuelve "nativo" de la situación o contexto que estamos analizando. Esa es la vida útil del fenómeno observado. Es un método interactivo de recopilación de información que requiere que el observador participe en los eventos o fenómenos observados. Participación significa participación social y puesta en común de las actividades básicas realizadas por los participantes de la formación. Lógicamente, dependiendo del objetivo del análisis, los datos recopilados o registrados serán de una u otra naturaleza para de esta manera aplicar los instrumentos a Docentes de la Universidad Nacional de Educación "UNAE" involucrados en el proceso de educación especial con mayor énfasis en el área de síndrome de Down.

\section{Identificacion de} problemas

1. Dificultades en el proceso de aprendizaje

2. Falta de programas de gamificación dirigidos a niños con Síndrome de Down

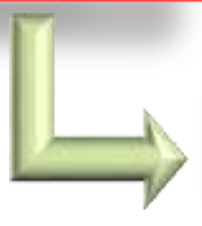

$$
\begin{aligned}
& \text { Formulación del 1. Propuesta de gamificación } \\
& \text { proyecto 2. Aprobación del proyecto }
\end{aligned}
$$
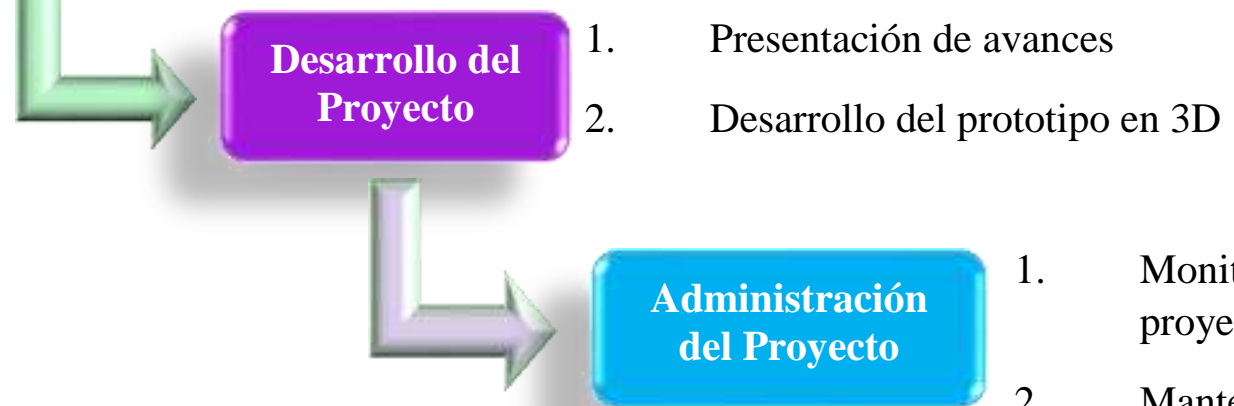

1. Monitoreo del proyecto

2. Mantenimiento del software

Implementación del proyecto

(Balarezo \& Mendieta, 2021) 


\section{RESULTADOS Y DISCUSIÓN}

En ecuador existe un aproximado de 18 programas de gamificación para niños basados en diferentes juegos como: comprensión lectora, matemáticas, memoria y lenguaje. Se ha implementado un total de 3 prototipos de gamificaciones dirigidos a niños con síndrome de Down en Ecuador.

Implementación de material educativo gamificado para la enseñanza-aprendizaje de la matemática en alumnos con Síndrome de Down, este estudio examina el impacto de la implementación de una propuesta didáctica en el aula basada en recursos educativos de juego (REG) sobre el rendimiento académico (RA) de niños / adolescentes con demencia, síndrome de Down, en problemas matemáticos; establecer estrategias metodológicas que faciliten el aprendizaje de los primeros conceptos matemáticos (conteos, conceptos de cantidades, suma y resta simple) y demostrar que el uso de estos recursos Los recursos educativos coloreados (juegos) y la metodología estructurada pueden mejorar la capacidad de los niños para aprender matemáticas. Al implementar recursos educativos gamificados, los estudiantes primero (antes) y último (después de la prueba) pasan por la prueba de Análisis de Habilidades Básicas para Comenzar (Riquelme, 2003). Esta prueba muestra el progreso de las habilidades, las técnicas y estadísticas utilizadas. Para analizar los datos fue el análisis de la varianza (ANOVA) y el análisis de estudiantes para comparar las puntuaciones medias. Más específicamente, los estudiantes con síndrome de Down después de analizar los resultados mostraron resultados de aprendizaje significativamente mejorados en el aprendizaje de los conocimientos y habilidades relacionados con la clasificación, socialización, preservación, demostrar juicio lógico y funciones simbólicas, aunque los mejores resultados están en habilidades de clasificación. , análisis lógico y juicio, no, en la expresión de función conservada y simbólica cuando se observa mejoría moderada (Nancy Castillo Jumbo \& José Jiménez González, 2020).

Software para apoyar el aprendizaje de fundamentos de programación basado en gamificación, El proyecto se desarrolló con el objetivo de apoyar la enseñanza y el aprendizaje de los fundamentos de la programación mediante la gamificación. Muchos estudiantes que toman cursos de programación tienen dificultades para aprender, lo que a veces conduce a la deserción. Por esta razón, es responsabilidad de las instituciones educativas y los docentes buscar constantemente nuevas estrategias y herramientas para mejorar los resultados del aprendizaje de los estudiantes. Si bien existen diferentes 
herramientas para enseñar programación, ninguna de ellas enfatiza el análisis y el cuestionamiento basado en el mismo bloque de código. El análisis de código es una actividad común para aprender a codificar y esto en una lección también se puede hacer en colaboración con el profesor responsable. Al intentar contribuir a este objetivo, se ha propuesto el desarrollo de una herramienta de software para ayudar a estudiantes y profesores en el proceso de enseñanza-aprendizaje de los fundamentos de la programación. Esto fue diseñado con la intención de contribuir a la inferencia lógica, fomentar las pruebas de escritorio y promover el análisis de código. Para ello, se utilizan actividades divertidas como contestar preguntas cronometradas, arreglar acertijos y algoritmos matemáticos mentales, "competir" con otros estudiantes. La propuesta comienza con la identificación de requisitos y lineamientos estéticos y funcionales a través de entrevistas y análisis de aplicaciones similares. Con esta instalación se construyó un prototipo, se evaluó su usabilidad. El software ha demostrado tener una amplia aceptación. En general, las puntuaciones de los estudiantes participantes asignados a las diferentes preguntas del cuestionario utilizado fueron altas (puntuaron una media de 15). Los profesores participantes también dieron críticas positivas sobre la utilidad del software. Estos resultados nos permiten deducir que la propuesta podría convertirse en una valiosa ayuda para aprender los fundamentos de la programación. Palabras clave: gamificación, enseñanza-aprendizaje, conceptos básicos de programación, tutoriales, requisitos, prototipos, usuarios representativos, evaluación de usabilidad (Rodríguez Neumane Moreta, 2020).

Desarrollo de un juego serio que ayude a las personas con síndrome de Down a mejorar sus competencias matemáticas, Hoy en día, con el aumento en el uso de computadoras y dispositivos móviles, hay muchas aplicaciones diferentes disponibles para personas con discapacidad. Sin embargo, se necesitan más aplicaciones en el campo de las matemáticas para las personas con síndrome de Down. El objetivo de este trabajo es desarrollar una aplicación para personas con síndrome de Down para apoyar el proceso de enseñanzaaprendizaje de la competencia matemática en el campo de las matemáticas: Crear correspondencias de "más que qué" y "menos que qué". Esta aplicación es esencialmente un juego accesible desde la web. Se realizó una revisión de la literatura sobre las características cognitivas y de aprendizaje de las personas con síndrome de Down y las características del software que se tuvieron en cuenta para el desarrollo de este tipo de 
aplicaciones. El desarrollo se realiza de acuerdo con el marco de Scrum. En este sentido, se realizaron seis Sprints. Y, en este documento, se detallan las herramientas y tecnologías utilizadas. Se realizaron pruebas con cinco estudiantes con síndrome de Down (con ejercicio leve a moderado) de la Fundación "Antorcha de Vida" para verificar el apoyo que el juego puede brindar en el desarrollo de sus habilidades matemáticas. Los resultados obtenidos fueron positivos ya que el $80 \%$ de los estudiantes logró progreso académico (Quinga \& Pastrano, 2018).

Diseño de un software para el aprendizaje lectoescritor en niños con síndrome de Down, la lectura y la escritura son habilidades que pueden verse como un proceso lógico que mejora el desarrollo cognitivo y hace frente a los desafíos que enfrentarán los estudiantes en un mundo globalizado, que se ve como débil, determinando el futuro de quienes serán hombres y mujeres. que hará de hoy un futuro mejor. Para desarrollar una estrategia de apoyo en el sector educativo, la investigación ha ayudado a identificar y analizar las fortalezas y debilidades de las poblaciones estudiantiles vulnerables; Una vez que es posible determinar el nivel alcanzado por los estudiantes en el proceso de enseñanza y aprendizaje, el software está diseñado para satisfacer las necesidades educativas que son fácilmente manejadas por todos los usuarios. VIII El diseño de software es una herramienta informática desarrollada con el propósito de mejorar el proceso de enseñanza y aprendizaje de la literatura, reuniendo características y aspectos funcionales, técnicos y pedagógicos como el reconocimiento, reconocimiento y sonido. Sonidos de fonemas, se almacena información específica en un base de datos. dirigido a estudiantes con síndrome de Down y con necesidades educativas del Centro de Educación Superior Básica No. 2 "Teodoro Wolf" ubicado en el estado de Santa Elena, provincia de Santa Elena (Jenny Rodriguez Lino, 2017).

\section{CONCLUSIÓN O CONSIDERACIONES FINALES}

Actualmente existen muchos métodos de enseñanza-aprendizaje en diferentes campos, especialmente el uso de escenarios virtuales y tecnologías, que ha reexaminado el uso de diferentes estrategias, métodos y materiales en el proceso de aprendizaje. Los recursos tecnológicos y dispositivos audiovisuales no siempre están al alcance de muchas personas o, en ocasiones, son difíciles de gestionar para padres y profesores. Por ello, se propone realizar una revisión sistemática centrada en la creación y posterior uso de prototipos de gamificación en enfoques de enseñanza-aprendizaje para la estimulación 
cognitiva en niños con síndrome de Down a partir de los 5-7 años. Según los diversos criterios recopilados en docentes que tienen especialidad en niños con Síndrome de Down se dedujo que el uso de las tecnologías está siendo actualmente una nueva forma innovadora de enseñar a los niños, en especial a los niños con esta condición, de tal forma que los niños aprenden de una manera más divertida y creativa. Se llegó a la conclusión que la propuesta de gamificación sería una buena opción para ayudar a la estimulación cognitiva de niños con Síndrome de Down, con juegos basados en percepción, atención, memoria, lenguaje, además de ser una herramienta a disposición de los docentes, padres de familia e instructores de niños con Síndrome de Down. En síntesis, es preciso mencionar que ningún tipo de discapacidad, es una barrera en la vida de cualquier ser humano, más bien es su fortaleza que lo hace mucho más fuerte, es por eso que la investigación derivado a un proyecto tiene como visión y misión mejorar la calidad educativa que se presentan tanto dentro como fuera de las aulas, el avance individual y personalizado para cada uno de los niños y niñas en el rango de edad de 5 a 7 años, llevando el significado de gamificación y educación hacia una nueva mirada tanto para padres, docentes, alumnos, instructores, instituciones educativas, familias y público en general.

\section{LISTA DE REFERENCIAS}

Consejo Nacional para la Igualdad de Discapacidades. (2021). Estadísticas de Discapacidad - Consejo Nacional para la Igualdad de Discapacidades. https://www.consejodiscapacidades.gob.ec/estadisticas-de-discapacidad/

EspertTortajada\&Villalba.

https://www.google.com/search?q=Espert+Tortajada\%2C+R.\%2C+\%26+Villalb a+Agustín\%2C+M.+D.+R.+(2014).+Estimulación+cognitiva\%3A+una+revisión +neuropsicológica.+Therapeia\% $2 \mathrm{C}+(6) \% 2 \mathrm{C}+73-$

93\&rlz=1C1ALOY_esEC952EC952\&oq=Espert+Tortajada\%2C+R.\%2C+\%26+ Villalba+Agustí

Federico Cintrón Fiallo. (2004). La división digital, los movimientos sociales y la educación. http://www.plazacritica.org/articulos04/divisiondigital04enlinea.pdf

Gerardo Meneses Benítez. (2016). El proceso de enseñanza- aprendizaje: el acto didáctico.

https://www.tdx.cat/bitstream/handle/10803/8929/Elprocesodeensenanza.pdf 
Jiménez, M. E. C. M. \& O. C. S. (2019). Inclusión educativa de las personas con necesidades educativas especiales permanentes. Universidad Técnica de Machala. http://scielo.sld.cu/scielo.php?pid=S1990$\underline{86442019000300195 \& \text { script }=\text { sci } \_ \text {arttext } \& \text { tlng }=\text { en }}$

José Flores Tena, M., \& Tena, F. (2021). Las nuevas tecnologías como estrategias innovadoras de enseñanza-aprendizaje en la era digital. Revista Electrónica Interuniversitaria de Formación Del Profesorado, 24(1), 29-42. https://doi.org/10.6018/REIFOP.406051

Kevin, C.-C., \& Cruces Geraldine. (2021). Importancia de la estimulación temprana para el desarrollo motor en niños con síndrome de Down: Una revisión sistemática. Revista Peruana de Ciencia de La Actividad Física y Del Deporte, 8(3), 10-10. https://doi.org/10.53820/RPCAFD.V8I3.152

Luis M. Echavarría-Ramírez, J. T.-U. (2021). Exploración neuropsicológica en niños con discapacidad intelectual. 11. https://www.researchgate.net/profile/LuisEchavarria-

$\underline{\text { Ramirez/publication/353343377_Exploracion_neuropsicologica_en_ninos_con_ }}$ discapacidad_intelectual/links/60f60c4cfb568a7098bffa11/Exploracionneuropsicologica-en-ninos-con-discapacidad-intelectual.pdf

Narvaja, M.-P. (2018). MINISTERIO DE CU LTURA y EDUCACIÓN Orientaciones para la elaboración de adecuaciones curriculares HACIA LAS ESCUELAS INCLUSIVAS. 164.

Ortiz Ocaña, A. (2013). Modelos Pedagógicos y Teorías del Aprendizaje. https://www.researchgate.net/publication/315835198

Pimienta, J. H. (2016). ESTRATEGIAS ENSEÑANZA-APRENDIZAJE ESTRATEGIAS DE ENSEÑANZA-APRENDIZAJE Estrategias de Enseñanza-Aprendizaje. 15.

Ruiz Rodríguez, E. (2009). Síndrome de Down: la etapa escolar. Guía para profesores y familias.

Vargas-Murillo, G. (2020). Estrategias educativas y tecnología digital en el proceso enseñanza aprendizaje. Cuadernos Hospital de Clínicas, 61(1), 114-129. http://www.scielo.org.bo/scielo.php?script=sci_arttext\&pid=S1652$\underline{67762020000100010 \& \operatorname{lng}=e s \& n r m=i s o \& t \operatorname{lng}=e s}$ 
Ximena Patricia Curay Correa, \& Diego Esteban Fernández Olivo. (2021). La gamificación como estrategia para motivar la lectura en el proceso enseñanza aprendizaje.

https://saber.ucab.edu.ve/xmlui/bitstream/handle/123456789/20042/Tendencias en investigación - Final \%281\%29.pdf?sequence=1\&isAllowed=y\#page=138

Jenny Rodriguez Lino. (2017). Diseño de un software para el aprendizaje lectoescritor en niños con síndrome de Down. https://repositorio.itb.edu.ec/bitstream/123456789/1441/1/PROYECTO DE GRADO DE RODRÍGUEZ LINO.pdf

Nancy Castillo Jumbo \& José Jiménez González. (2020). Vista de Implementación de material educativo gamificado para la enseñanza-aprendizaje de la matemática en alumnos con Síndrome de Down. https://revistas.um.es/riite/article/view/397741/283041

Quinga, J. M., \& Pastrano, M. (2018). Desarrollo de un juego serio que ayude a las personas con síndrome de Down a mejorar sus competencias matemáticas. http://bibdigital.epn.edu.ec/handle/15000/19599

Riquelme, G. (2003). TEST DE HABILIDADES BÁSICAS PARA LA INICIACIÓN AL CÁLCULO “TIC” BASIC ABILITIES FOR CALCULUS INTRODUCTION TEST “TIC. ” 20.

Rocío, \& Peredo Videa, A. (2016). Comprendiendo la discapacidad intelectual:: datos, criterios y reflexiones. Revista de Investigacion Psicologica, 15(15), 101-122. http://www.scielo.org.bo/scielo.php?script=sci_arttext\&pid=S222330322016000100007\&lng=es\&nrm=iso\&tlng=es

Rodríguez Neumane Moreta, R. E. (2020). UNIVERSIDAD TÉCNICA ESTATAL DE QUEVEDO FACULTAD DE CIENCIAS DE LA INGENIERÍA.

Zúñiga, M. R. R., Hurtado, G. P. P., \& Pérez, J. M. (2020). La capacidad de la gestión de la configuración en las pequeñas organizaciones de desarrollo de software. ConcienciaDigital, $3(3)$, 466-485. https://doi.org/10.33262/concienciadigital.v3i3.1339 